\title{
Interferon alfa-2b for decompensated liver disease caused by either chronic hepatitis B or C: preliminary results of a pilot study
}

\author{
M Dimopoulou, K Fafoutis, K Basiliou, J Ketikoglou, G Karvountzis
}

State Department of Medicine, Hippokration Hospital, Athens, Greece M Dimopoulou K Fafoutis K Basiliou J Ketikoglou G Karvountzis Correspondence to: Dr G Karvountzis, Medicine, Hippokration Hospital, Athens, Greece. State Department of

\begin{abstract}
This pilot study was designed to assess the tolerance and effectiveness of interferon alfa-2b in patients with decompensated liver disease caused by chronic hepatitis $B$ $(n=7)$ or $C(n=5)$. Preliminary results suggest that patients may benefit from small doses of interferon (improvement in liver function tests and reduction in alanine aminotransferase), although the decreased tolerance and dose dependent increase in side effects in these patients with liver disease underlines the need for close follow up and the dose adjustment of interferon treatment.
\end{abstract}

(Gut 1993; supplement: S104-S105)

TABLE I Patient characteristics

\begin{tabular}{lcc}
\hline & Hepatitis $B$ & Hepatitis $C$ \\
\hline Male/female & $6 / 1$ & $1 / 4$ \\
Mean age (y) (range) & $51(46-65)$ & $57(47-69)$ \\
Anti-HCV positive & 0 & 5 \\
HBsAg positive/HBV-DNA positive & $7 / 7$ & 0 \\
HBeAg positive/anti-HBe positive & $1 / 6$ & 0 \\
Presence of ascites & 7 & 5
\end{tabular}

TABLE II Mean laboratory data before and at the end of treatment in $\mathrm{HBs} A g$ positive patients

\begin{tabular}{lcc}
\hline & Before treatment & End of treatment \\
\hline Alanine aminotransferase (IU/l) & 270 & 80 \\
Bilirubin (mg/dl) & $3 \cdot 8$ & $1 \cdot 8$ \\
Prothrombin time (s) & 21 & 18 \\
White blood count $\left(/ \mathrm{mm}^{3}\right)$ & 5200 & 2600 \\
Platelets $\left(/ \mathrm{mm}^{3}\right)$ & 130000 & 60000 \\
HBV-DNA $(\mathrm{pg} / \mathrm{ml})$ & 39 & $1 \cdot 4$ \\
\hline
\end{tabular}

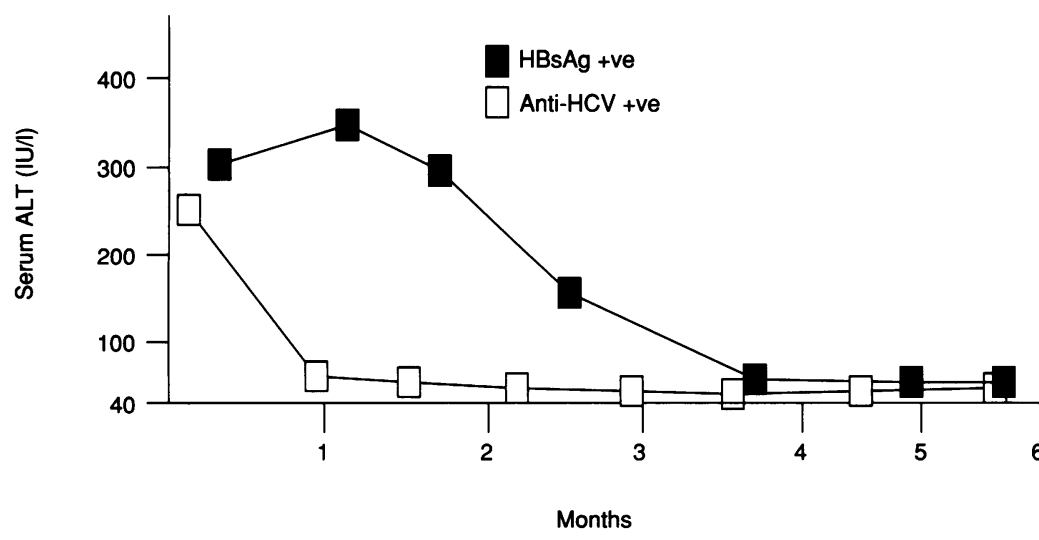

Figure Alanine aminotransferase changes in 12 patients with decompensated liver disease caused by chronic hepatitis $B$ and $C$.
The aim of this pilot study was to assess the tolerance and effectiveness of interferon alfa$2 b$ (INTRON A) in patients with decompensated liver disease caused by chronic hepatitis B or C. This study gives preliminary results for 12 patients.

\section{Patients and methods}

Patients were required to have decompensated liver disease proved by biopsy examination (the criteria used to define decompensated liver disease in this study included the presence of ascitis, serum albumin values below $3 \mathrm{~g} / \mathrm{dl}$, and serum bilirubin values above $3 \mathrm{mg} / \mathrm{dl}$ ) (positive for either hepatitis B surface antigen ( $\mathrm{HBsAg}$ ) or antibodies to hepatitis $\mathrm{C}$ virus (anti-HCV)), serum transaminases 2.5 times the upper limit of normal for more than six months, and absence of encephalopathy, active bleeding, renal failure, or detectable hepatocellular carcinoma.

Interferon alfa- $2 \mathrm{~b}$ was given percutaneously three times weekly for four months. The initial dose was 1 million units (MU) for 10 days and could be increased up to $3 \mathrm{MU}$ if clinical and laboratory investigation did not show serious side effects. Examination of all patients was performed every 10 days for the first month, and monthly thereafter. Efficacy end points were taken as liver function tests, white blood cell and platelet counts, and clinical status.

\section{Preliminary results}

Of the 12 patients included in the study, seven had hepatitis B and five had hepatitis C (Table I). Mean laboratory data before and at the end of interferon treatment are shown in Tables II and III.

Liver function tests tended to show improvement during treatment, with a significant reduction in serum alanine aminotransferase activities in both $\mathrm{HBsAg}$ positive and anti-HCV positive patients, although the pattern of response varied between the two disease types (Figure). Hepatitis C patients showed a steady decrease in alanine aminotransferase activities, while hepatitis B patients had a slight increase in alanine aminotransferase activities before they declined, as seen in those who responded to treatment in other studies using interferon in chronic hepatitis B.

One patient with $\mathrm{HBeAg}$ positive cirrhosis and massive ascitis experienced a biochemical 
TABLE III Mean laboratory data before and at the end of treatment in anti-HCV positive patients

\begin{tabular}{lcc}
\hline & Before treatment & End of treatment \\
\hline Alanine aminotransferase (IU/l) & 230 & 60 \\
Bilirubin (mg/dl) & $2 \cdot 8$ & $1 \cdot 8$ \\
Prothrombin time (s) & 21 & 17 \\
White blood cells $\left(/ \mathrm{mm}^{3}\right)$ & 5100 & 2300 \\
Platelets $\left(/ \mathrm{mm}^{3}\right)$ & 110000 & 65000 \\
\hline
\end{tabular}

flare up with clinical worsening (encephalopathy) while receiving $2 \mathrm{MU}$ during the second week of treatment. The dose was reduced to 1 $\mathrm{MU}$ and, ultimately, seroconversion of $\mathrm{HBeAg}$ to anti-HBeAg was seen, accompanied by biochemical remission and loss of HBsAg with appearance of anti-HBs.

All patients reported appreciable clinical improvement in terms of weakness, appetite, and general feeling. But, in the study group of patients with decompensated cirrhosis caused by chronic hepatitis $\mathrm{B}$ and $\mathrm{C}$, only doses of interferon less than $2 \mathrm{MU}$ three times weekly could be tolerated. Thrombocytopenia (platelets $<50000 / \mathrm{mm}^{3}$ ) was the most common complication.

\section{Conclusions}

These preliminary results indicate that patients with decompensated liver disease caused by chronic hepatitis $B$ and $C$ can benefit from small doses of interferon alfa-2b. The decreased tolerance and the dose dependent incidence of side effects of interferon in these patients emphasises the need for close follow up and dose adjustments. 